\title{
Erratum zu: Weiterbildung Suchttherapie- Psychodrama (DRV-anerkannt) am Moreno Institut Stuttgart
}

\author{
Wolfgang Treiber
}

\section{Erratum zu: Z Psychodrama Soziometr (2013) 11 (Suppl 1):223-226 DOI: $10.1007 / s 11620-012-0173-6$}

Im Supplement 1/Sonderheft 4 der Zeitschrift für Psychodrama und Soziometrie „Das Drama der Abhängigkeit“ wurde die Weiterbildung „Suchttherapie-Psychodrama (DRVanerkannt) in Deutschland“ vom Autor zum Teil missverständlich und auch inhaltlich nicht ganz der Sachlage entsprechend dargestellt. Wir bedauern dies und veröffentlichen nachfolgend die korrekte Beschreibung der Weiterbildung „Suchttherapie-Psychodrama (DRV-anerkannt) in Deutschland“. Die Darstellung haben drei VertreterInnen der Weiterbildungsgänge übernommen: Dorothea Ensel (Moreno-Institut Stuttgart), Stefan Flegelskamp (Psychodrama Institut Szenen), Helmut Schwehm (fdr und Moreno-Institut Goslar/Überlingen).

\section{Weiterbildung Suchttherapie-Psychodrama (DRV-anerkannt) in Deutschland}

Dorothea Ensel, Stefan Flegelskamp, Helmut Schwehm

Die postgraduierte, berufsbegleitende Weiterbildung „Suchttherapie-Psychodrama“ umfasst eine Dauer von 3 Jahren und wird für Fachkräfte aus dem Arbeitsfeld Sucht in Deutschland von den drei Trägern nach den Richtlinien der Deutschen Rentenversicherung (DRV) angeboten und durchgeführt: Fachverband Drogen und Suchthilfe (fdr) in

\footnotetext{
Online publiziert: 03.04 .2013

(C) Springer Fachmedien Wiesbaden 2013 doi:http://dx.doi.org/10.1007/s11620-012-0173-6

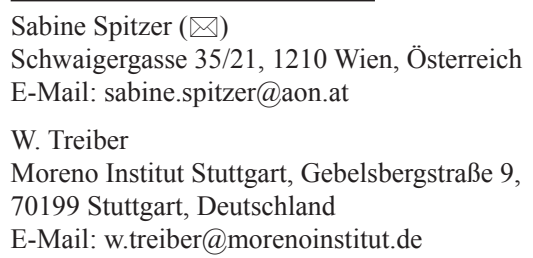

Die Onlineversion des Originalbeitrages ist erreichbar unter 
Kooperation mit dem Moreno Institut Goslar/Überlingen, Moreno Institut Stuttgart und dem Psychodrama Institut Szenen in Köln. Die WeiterbildungsleiterInnen und SupervisorInnen der jeweiligen Institute verfügen über fundierte Kenntnisse in der Methode Psychodrama, sowie über mehrjährige fachspezifische Berufserfahrung im Tätigkeitsfeld Sucht.

Die Weiterbildung beinhaltet die Vermittlung methodischer und theoretischer Kenntnisse des Psychodramas und seiner Anwendung im Praxisfeld Rehabilitation, Sucht, die methoden- geleitete psychodramatische Selbsterfahrung und die supervidierte eigenständige Anwendung psychodramatischer Kenntnisse im Praxisfeld. Die Weiterbildung umfasst folgende integrale Bestandteile: 1) Selbsterfahrung, 2) Theorie, 3 ) Themenzentrierte Weiterbildungseinheiten, 4) Fallpraxis, 5) Supervision (Einzel- und Gruppensupervision), 6) Berufsbegleitende Trainingsgruppe, 7) Peergroups, 8) Selbststudium.

Die dreijährige Weiterbildung gliedert sich in Grundstufe und Anwendungsstufe. Spezifische Themen der Weiterbildung sind:

- Einführung in die psychodramatische und inhaltliche Arbeit der Oberstufe

- Ätiologie, Erklärungs- und Behandlungsmodelle von Süchten

- Anamnese, Diagnostik, Indikationsstellung und Motivation in der Initialphase der Therapie

- Arbeit mit Einzel- und Gruppensetting sowie in Kombination beider Settings

- Aspekte des Behandlungsprozesses und die Rolle des/der Therapeuten/Therapeutin

- Arbeit mit sozialen Bezugssystemen

- Spezifische Aspekte der Suchttherapie (z. B. Gender, Behandlung von nicht stoffgebunden Süchten, Indikationen für unterschiedliche Behandlungsformen)

- Spezifische Krisen und Rückfall/Rückfallprävention in der Suchtbehandlung

Die Weiterbildung schließt mit einer Prüfung ab. Wissenschaftliche Hausarbeit, schriftliche Klausur und Colloquium sind Bestandteile der Prüfung.

Nach Abschluss der Prüfung und Erhalt des Zertifikats sind die AbsolventInnen als SuchttherapeutIn-Psychodrama (DRV-anerkannt) befähigt, in der ambulanten, ganztägig ambulanten und stationären medizinischen Rehabilitation Sucht als Einzel- und GruppentherapeutIn zu arbeiten. Bereits mit Beginn der Weiterbildung ist es möglich, als Co-TherapeutIn tätig zu sein.

Als Zulassungsvoraussetzung für die Teilnahme an der Weiterbildung gilt gemäß der Vereinbarung „Abhängigkeitserkrankungen“ vom 04.05.2001 eine abgeschlossene Ausbildung als Arzt/Ärztin (1. und 2. Staatsexamen), Dipl. PsychologIn (Diplom oder Master), Dipl.-SozialarbeiterIn und Dipl.-SozialpädagogIn (Diplom oder Bachelor). Zu den Zulassungsvoraussetzungen gehört auch die Prüfung der persönlichen Eignung. Für die Dauer der Weiterbildung muss ein Arbeitsplatz mit dem zeitlichen Umfang von mindestens 50\% der wöchentlichen Regelarbeitszeit im Praxisfeld der Suchtkrankenhilfe nachgewiesen werden. Die DRV hat 2012 die Kriterien für die Anerkennung von Weiterbildungsangeboten für das Tätigkeitsfeld neu formuliert und veröffentlicht.

Ausführliche Informationen zur Weiterbildung sowie eine detaillierte Ausführung zu den Zugangsvoraussetzungen sind auf den Homepages aller drei Träger zu finden. Anmeldungen zur Weiterbildung Suchttherapie-Psychodrama (DRV-anerkannt) sind dort ebenfalls möglich. 
fdr fachverband drogen und Moreno Institut Stuttgart suchthilfe

Odeonstraße 14

30159 Hannover

$0511 / 18333$

www.fdr.online.info

in Kooperation mit

Moreno Institut

Goslar-Überlingen

$05321 / 319317$

www.moreno-goslar-über-

lingen.de
Gebelsbergstr. 9

70199 Stuttgart

0711/606707

www.morenoinstitut.de
Psychodrama Institut Szenen

Barbarossaplatz 7

50674 Köln

0221/67789352

www.szenen.de 